\title{
Minutenschnelle Verseifung von Lipiden bei Raumtemperatur
}

\author{
Von P. Hušek, Marta Králíková und V. Felt \\ Aus dem Forschungsinstitut für Endokrinologie (Direktor: Doz. MUDr. K. Šilink, Dr.Sc.) Prag, Tschecboslow'akci
}

(Eingegangen am 21. Mai/7. September 1973)

\begin{abstract}
Aufgrund einer stark exothermischen Reaktion zwischen einem Teil Chloroform und zwei Teilen gesättigter methanolischer Kalilaugelösung lassen sich Phosphoglyceride, Glyceride und in kleineren Mengen auch Cholesterinester innerhalb von einigen Minuten praktisch völlig verseifen. Durch gaschromatographische Untersuchung der Lipidfettsäuren wird die Vollständigkeit der Verseifung von einzelnen Serumlipiden dargestellt.
\end{abstract}

\section{Saponification of lipids within a few minutes at room temperature}

A rapid, exothermic reaction between one part of chloroform and two parts of saturated methanolic potassium hydroxide solution leads to an almost complete saponification of phosphoglycerides, glycerides and small amounts of cholesterol esters within a few minutes. The complete course of the saponification of each serum lipid was confirmed by the gas chromatographic cstimation of fatty acids.

Früher hat man geglaubt (1), daß die alkalisch katalysierte Umesterung (Alkoholyse) und die Verseifung (Hydrolyse) von Lipiden sich grundsätzlich unterscheiden. Während bei der Alkoholyse das Alkali als Alkoholat nur zur Katalysierung der Umesterungsreaktion dient und man unter sorgfältigem Wasserausschluß arbeitete, setzt sich bei der Hydrolyse die $\mathrm{Na}$ tron- oder Kalilauge unter Salzbildung mit den Fettsäuren um. Ein geringer Wasserzusatz, glaubte man, führt zur eindeutigen Verlagerung des Reaktionsgleichgewichtes zur Seifenbildung hin.

In den letzten Jahren wurde ein klarer Beweis dafür erbracht, $\mathrm{da} \beta$ in alkoholischen Laugelösungen die basenkatalysierte Umesterung und die durch Lauge bewirkte Verseifung in einem engen Zusammenhang stehen $(2,3)$. Ein Wasserausschluß bei dem Umesterungsvorgang hat sich nicht als erforderlich bestätigt, denn die schwach alkalischen alkoholischen Laugelösungen setzen die Lipide in die Fettsäureesterform gleich wirksam um wie die Alkoholsalze, die durch Auflösung von Metall in wasserfreiem Alkohol hergestellt werden (4-7). Darüber hinaus wird das Reaktionsgleichgewicht nicht besonders durch Wasserzusatz, sondern viel mehr durch die Laugenkonzentration und die Zeitdauer auf die Seite der Verseifungsprodukte verschoben.

Zwei Feststellungen sind heute unbestritten:

a) Bei jeder Verseifung tritt die Umesterung als erste Stufe ein, d. h. daß nicht die Fette' direkt, sondern erst die gebildeten alkoholischen Fettsäureester verseift werden. Dadurch läßt sich auch erklären, warum die Verseifung in alkoholischen Laugelösungen viel schneller vor sich geht als in wäßriger Lösung.

b) Die Verseifung von Estern, der zweite Reaktionsschritt, verläuft im Vergleich mit der Umesterung viel langsamer. Der relative Unterschied in den beiden Reaktionsgeschwindigkeiten wurde als Faktor 1500 ermittelt (8). Die Erklärung dafür, daß die Alkoholyse der Hydrolyse vorangeht, ist darin zu suchen, daß auch in Anwesenheit erheblicher Wassermengen die überwiegende Form in den alkoholischen Laugelösungen nicht Hydroxyl- sondern Alkoxydat-Ionen sind. In methanolischen Lösungen, die einige Prozent Wasser enthalten, wird praktisch das Gesamthydroxyd in die Methoxydform umgesetzt. Das Gleichgewicht der Reaktion

$$
\mathrm{NaOH}+\mathrm{CH}_{3} \mathrm{OH} \rightleftharpoons \mathrm{CH}_{3} \mathrm{ONa}+\mathrm{HOH} \text { (Gl. 1) }
$$

liegt beinahe völlig auf der Seite der Reaktionsprodukte. Im ersten Reaktionsschritt wirkt demnach die Lauge nur als Katalysator und das Lipid wird innerhalb von Sekunden umgeestert. Die Reaktion ist umkehrbar:

$\mathrm{R}_{1} \mathrm{COOR}_{2}+\mathrm{CH}_{3} \mathrm{ONa} \rightleftharpoons \mathrm{R}_{1} \mathrm{COOCH}_{3}+\mathrm{R}_{2} \mathrm{ONa}$

Im zweiten Reaktionsschritt werden die alkoholischen Ester durch die überschüssige Lauge langsam zu den Fettsäuresalzen hydrolysiert. Diese Reaktion, im Gegensatz zu der ersten, ist nicht umkehrbar:

$\mathrm{R}_{1} \mathrm{COOCH}_{3}+\mathrm{NaOH} \rightarrow \mathrm{R}_{1} \mathrm{COONa}+\mathrm{CH}_{3} \mathrm{OH}$

Aus der letzten Reaktionsgleichung (Gl. 3) ist ersichtlich, daß man ständig einen Laugeüberschuß haben muß, wenn man die Reaktion eindeutig auf die Seite der Verseifungsprodukte verschieben will. Die Lauge wird durch Umkehrung der Reaktion 1 gewonnen, was wieder genug Wasser im Reaktionssystem erfordert. Dies wurde bisher durch drei verschiedene Verfahren erreicht:

1. Wasserzugabe: $5 \mathrm{ml} \mathrm{H}_{2} \mathrm{O} / 100 \mathrm{ml}$ methanolischer (1 mol/l) Natronlaugelösung, Verseifungsdauer 60 bis $90 \mathrm{~min}$ bei $70-80^{\circ} \mathrm{C}$ (älterer Bericht) (1); $0,25 \mathrm{ml}$ $\mathrm{H}_{2} \mathrm{O} / 100 \mathrm{ml}$ methanolischer $(0,5 \mathrm{~mol} / \mathrm{l})$ Natronlaugelösung, $30 \mathrm{~min}$ bei Raumtemperatur (versucht an Trilinolein (3)), 
2. Erhöhung der Laugekonzentration und damit auch des Wassergehaltes: bis $9 \mathrm{~mol} / \mathrm{l}$ konzentrierte alkoholische Laugelösungen und Reaktionszeiten von $12-24$ h (9),

3. "Aktivierung" von Reaktionswasser durch Erwärmen: $0,5 \mathrm{~mol} / 1$ äthanolische Kalilauge, 20 bis $30 \mathrm{~min}$ bei $55-70^{\circ} \mathrm{C}$ (für Serumglyceride (10)).

Das Ganze stellt ein breites Spektrum von milderen bis zu drastischen Verseifungsbedingungen dar, wobei ein klares eindeutiges Verseifungsverfahren schwierig $\mathrm{zu}$ finden ist. Bei der Gewinnung von Fettsäuren aus Fett oder bei der Bestimmung von einzelnen Lipidgruppen mittels Titration der aus ihnen freigesetzten Fettsäuren verzögert der Verseifungsvorgang das ganze Verfahren erheblich; gewöhnlich wird auch eine Energiequelle für das Erwärmen der Probe erforderlich. Das hier dargestellte Verfahren nutzt die freigesetzte Wärme der Chloroform-Kaliummethylat-Reaktion aus, wobei sich die Lipide schnell und praktisch quantitativ innerhalb weniger Minuten verseifen lassen.

\section{Material und Methoden}

\section{Reagenzien :}

Zur Verseifung von Lipiden: gesättigte methanolische Kalilaugelösung (etwa $40 \mathrm{~g} \mathrm{KOH}$ in $100 \mathrm{ml}$ Methanol lösen, so daß am Boden noch einige Plätzchen übrigbleiben; mindestens $24 \mathrm{~h}$ „reifen" lassen); Chloroform p. a. (Lachema, Brno), zur Extraktion und Titration von Fettsäuren (11): Heptan p. a., Isopropanol p. a., $0,25,0,5$ und $5 \mathrm{~mol} / 1 \mathrm{H}_{2} \mathrm{SO}_{4}$; Natriumsulfat $\left(\mathrm{Na}_{2} \mathrm{SO}_{4} \cdot 10 \mathrm{H}_{2} \mathrm{O}\right)$; $0,02(0,2) \mathrm{mol} / 1 \mathrm{NaOH}$; Nil-Blau Indikator, $10 \mathrm{~g} / \mathrm{l}$ alkohol. Lösung (Wasser: abs. Äthanol $=50 \mathrm{ml}+950 \mathrm{ml}$ ).

Lipid-Standards: Glycerin-Tripalmitat (Brit. Drug Houses Ltd.), L- $\alpha$-Lecithin, Cholesterylpalmitin, -stearin, -olein, -linolein und -linolenin (Koch Light Labor. Ltd.), alle Chemikalien in Reinheit „pure“.

\section{Methodik}

$\mathrm{Zu}$ den in $1 \mathrm{ml}$ Chloroform gelösten Lipiden im Reaktionsgefäß (Reagenzglas $15 \mathrm{~cm}$, Schliff 19/26) gibt man langsam $2 \mathrm{ml}$ gesättigter methanolischer Kalilauge. Nach Zugabe von etwa $0,5 \mathrm{ml}$ fängt das Chloroform-Methanol-Kalilauge Gemisch stürmisch an zu sieden, wobei das entstehende Kaliumchlorid an die Wände spritzt. Nachdem die gesamten $2 \mathrm{ml}$ zugesetzt wurden, läßt man die Reaktion noch 15 min verlaufen. Dann wird sie durch Zugabe von $5 \mathrm{ml} 0,25 \mathrm{~mol} / \mathrm{l} \mathrm{H}_{2} \mathrm{SO}_{4}$ beendet, wobei gleichzeitig der Laugeüberschuß neutralisiert und das ganze Gemisch angesäuert wird. Die freien Fettsäuren lassen sich nach dem TrourVerfahren (11) extrahieren und titrimetrisch bestimmen: es wird mit $5 \mathrm{ml} \mathrm{Heptan} \mathrm{und} 5 \mathrm{ml}$ Extraktionsgemisch (Isopropranol/ Heptan $/ 1 \mathrm{~mol} / 1 \mathrm{H}_{2} \mathrm{SO}_{4}=40 \mathrm{ml}+10 \mathrm{ml}+1 \mathrm{ml}$ ) geschüttelt, dann wird die Heptanschicht (mit den Fettsäuren) abgezogen und in einem zweiten Reagenzglas mit dem Ausschüttelgemisch (11) $\left(22,7 \mathrm{~g} \mathrm{Na}_{2} \mathrm{SO}_{4} \cdot 10 \mathrm{H}_{2} \mathrm{O}\right.$ und $2 \mathrm{ml} 10 \mathrm{~mol} / 1 \mathrm{H}_{2} \mathrm{SO}_{4}$ mit Wasser auf $1000 \mathrm{ml}$ ) behandelt. Ein Aliquot wird zur Titration eingesetzt.

\section{Ergebnisse}

In Tabelle 1 wird der zeitliche Verlauf der Reaktion zwischen Chloroform und gesättigter methanolischer Kalilaugelösung angezeigt. Gleichzeitig wird durch diese Werte die Richtigkeit des Mischverhältnisses eins zu zwei (Chloroform/alkoholische Lauge), so wie es aus der Reaktion 4 (siehe Diskussion) hervorgeht, bestätigt.
Tab. 1

Zeitlicher Verlauf der Reaktion zwischen Chloroform ( $1 \mathrm{ml}$ ) und gesättigter methanolischer Kalilaugelösung (2 $\mathrm{ml}$, langsam zugeben). Anfangskonzentration der Kalilauge im Methanol (gesättigt bei $20-25^{\circ} \mathrm{C}$ ): $6,90-7,05 \mathrm{~mol} / 1$ (etwa $39 \mathrm{~g} / 100 \mathrm{ml}$ )

\begin{tabular}{ccc}
$\begin{array}{c}\text { Zeit nach } \\
\text { Vermischen beider } \\
\text { Reagenzien } \\
\text { [min] }\end{array}$ & Masse-Fraktion & mol/1 \\
\hline sofort & 0,22 & 1,44 \\
2 & 0,14 & 0,98 \\
4 & 0,11 & 0,77 \\
8 & 0,07 & 0,49 \\
10 & 0,06 & 0,42 \\
15 & 0,06 & 0,42 \\
60 & 0,05 & 0,35 \\
\hline
\end{tabular}

Abbildung 1 zeigt den zeitlichen Verlauf der Verseifung von Glyceriden (Tripalmitin) und Phosphoglyceriden (Lecithin) an. Um die Richtigkeit der Verseifungstheorie klarzustellen, wurde auch der Gehalt an Fettsäuremethylestern, nachdem die Reaktion nach verschiedenen Zeitintervallen gestoppt wurde, gaschromatographisch bestimmt.

Um den oberen Wert des Konzentrationsbereiches, bei dem man noch mit praktisch vollständiger Verseifung rechnen kann, zu ermitteln, wurden verschiedene Mengen von Glyceriden, Phosphoglyceriden und Cholesterinestern in $1 \mathrm{ml}$ Chloroform gelöst und verseift. Aus der Abbildung 2 ist klar ersichtlich, bis zu welcher Konzentration das Verseifungsverfahren noch wirksam ist.

Um die Vollständigkeit der Hydrolyse von einzelnen Fettsäuren nachzuprüfen, wurden Serumlipide durch zwei unabhängige Verfahren untersucht. Dưrch Verseifung freigesetzte Fettsäuren wurden wieder mit $\mathrm{BF}_{3^{-}}$ Methanol verestert $(12,13)$ und die Ergebnisse mit

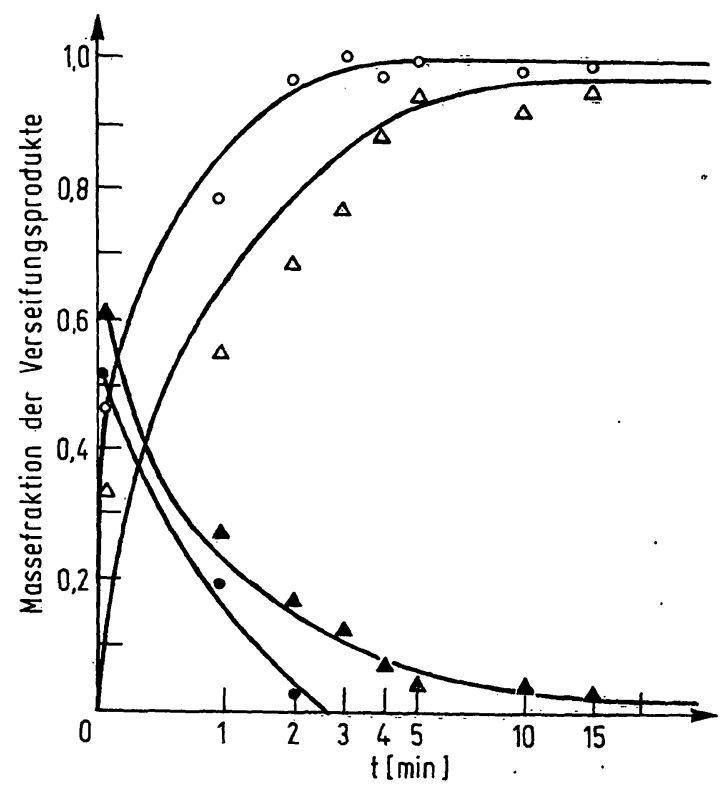

Abb, 1

Verseifungsgeschwindigkeit (min) von Glyceriden ( $5 \mathrm{mg}$ Tripalmitin $\Delta,{ }_{\text {der }}$ Bestimmung der entstandenen Produkte in verschiedenen ZeitOffene Symbole: Säure intervallen

Offene Symbole: Säure; geschlossene Symbole: Methylester 
Tab. 2

Untersuchung zur Wirksamkeit des beschriebenen Verseifungsverfahrens für alle in Serumlipiden vorkommenden Fettsäuren. Die einzelnen Serumlipidfraktionen wurden entweder alkalisoh umgeestert oder hydrolysiert und nachfolgend mit $B F_{3}-M_{e}$ thanol verestert. Die Werte stellen den Umkehrwert aus je drei Bestimmungen ein und desselben Serums dar

\begin{tabular}{|c|c|c|c|c|c|c|}
\hline \multirow[b]{2}{*}{ Fettsäure } & \multicolumn{2}{|c|}{ Phosphoglyceride } & \multicolumn{2}{|c|}{ Triglyceride } & \multicolumn{2}{|c|}{ Cholesterinester } \\
\hline & Umesterung & Hydrolyse & Umesterung & Hydrolyse & Umesterung & Hydrolyse \\
\hline $14: 0$ & - & - & 1,4 & 1,5 & - & - \\
\hline $16: 1$ & 2,8 & 2,8 & 5,2 & 5,4 & 5,4 & 5,6 \\
\hline $18: 0$ & 17,0 & 17,6 & 4,1 & 4,6 & 2,0 & 2,4 \\
\hline $18: 1$ & 18,3 & 18,8 & 48,3 & 47,8 & 27,3 & 25,5 \\
\hline $18: 2$ & 15,9 & 15,5 & 12,4 & 11,6 & 43,5 & 43,2 \\
\hline $20: 4$ & 11,3 & 10,8 & - & - & 7,7 & 8,0 \\
\hline ntmenge $(\mathrm{g} / \mathrm{l})$ & 2,23 & $\left.(1,80)^{*}\right)$ & 2,40 & 2,28 & 2,07 & 1,97 \\
\hline
\end{tabular}

*) Die Phosphoglyceride wurden nicht eluiert, sondern direkt auf dem Kieselgel hydrolysiert

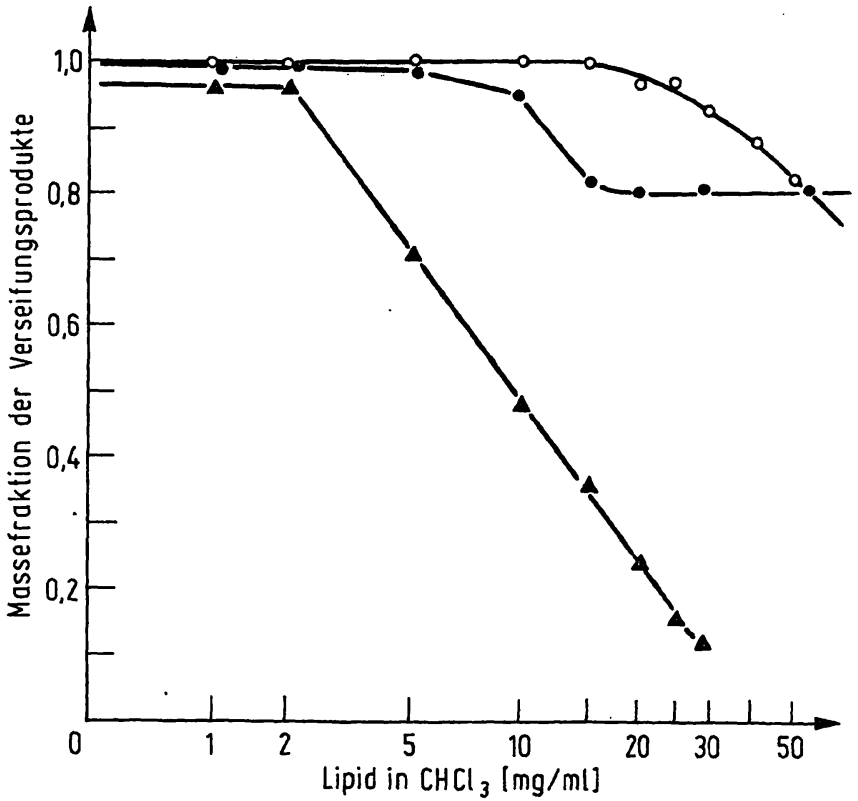

Abb. 2

Verseifungsausbeute bei Verwendung verschiedener Ausgangsmengen von Lipiden in $1 \mathrm{ml}$ Chloroform. Untersucht an Tripalmitin (-- - ) L- $\alpha$-Lecithin (O-O) und einem Gemisch von Cholesterinestern zeit $15 \mathrm{~min}$

denen verglichen, die durch die basenkatalysierte Umesterungsmethode einzelner Lipidgruppen mit $0,5 \mathrm{~mol} / \mathrm{l}$ Natriummethylat gewonnen wurden (7). Die Fettsäuremethylester wurden gaschromatographisch (DEGSSäule, FID) bestimmt. Der Gehalt an einzelnen Fettsäuren, in Masse-Fraktionen $(\mathrm{g} / \mathrm{g} \times 100)$ dargestellt, der durch die zwei Verfahren ermittelt wurde, geht aus der Tabelle 2 hervor.

\section{Diskussion}

Um die Reaktion zwischen Chloroform und gesättigter methanolischer Kalilaugelösung (Kaliumhydroxyd wurde wegen seiner größeren Löslichkeit im Alkohol bevorzugt) zu ermitteln, haben wir sie mit verschiedenen Mengen von beiden Reagenzien untersucht. Das sich bei der Reaktion bildende Kaliumchlorid wird teilweise an die Wände verspritzt, zum größeren Teil setzt es sich jedoch zusammen mit dem Wasser und dem Methanolüberschuß auf dem Boden des Reaktionsgefäßes ab.
Aus dem Chloroform entsteht außer Kaliumchlorid noch ein flüchtiges Produkt, vermutlich Dichlormcthylmethyläther, so daß man das stächiometrische Mischungsverhältnis Chloroform vs. gesättigte methanolische Kalilauge, $1: 2$, aus dem Reaktionsschema errechnen kann (Methanol befindet sich im Überschuß):

$\mathrm{CHCl}_{3}+\mathrm{CH}_{3} \mathrm{OH}+\mathrm{KOH} \rightarrow \mathrm{KCl}+\mathrm{H}_{2} \mathrm{O}+$ $\mathrm{CHCl}_{2}-\mathrm{O}-\mathrm{CH}_{3}$

Die restliche Alkalität wurde nach verschiedenen Zeitintervallen titrimetrisch gemessen. Aus Tabelle 1 geht hervor, daß bei dem gewählten Mischungsverhältnis unmittelbar nach der Zugabe der methanolischen Kalilauge $1 / 5$ der gesamten Alkalität durch Chloroform neutralisiert werden. Darüber hinaus sinkt schon nach einigen Minuten die Laugekonzentration auf etwa $0,5 \mathrm{~mol} / 1$, was einen günstigen Laugeüberschuß für die nachfolgende Verseifung darstellt und zugleich die Gefahr einer eventuellen Isomerisierung von ungesättigten Fettsäuren verringert (siehe auch Tab. 2).

Aus Abbildung 1 wird deutlich, daß die Verseifung von Lipiderı in alkoholischen Laugelösungen über die Methylesterbildung geschieht und $\mathrm{da} \beta$ die Hydrolyse langsamer verläuft. Doch wird schon eine Minute nach dem Mischen beider Reagenzien mehr als die Hälfte der Lipide verseift! Die Fettsäuremethylester werden während der Zugabe gebildet (die vorsichtige langsame Zugabe von alkoholischer Kalilauge nimmt etwa eine Minute in Anspruch) und gleich weiter verseift. Bemerkenswert ist ebenfalls die Tatsache, daß sich die Phosphoglyceride schneller und vollkommener verseifen lassen, während die Glyceride noch Spuren von Methylestern enthalten. Die Einfachheit der Verseifung von Phosphoglyceriden wird auch durch Abbildung 2 bestätigt, wo man bis zu einer Konzentration von $20 \mathrm{mg} / \mathrm{ml}$ Chloroform mit fast vollkommener Verseifung rechnen kann. Die Glyceride und Cholesterinester werden nur bis zu einem Gehalt von $5-10 \mathrm{mg} / \mathrm{ml}$ bzw. $2 \mathrm{mg} / \mathrm{ml}$ völlig verseift. Eine Erklärung dafürjist von rein theoretischem Charakter; die an Glycerin esterartig gebundene Phosphorsäure wird durch die Lauge unmittelbar abgespalten, wodurch die ganze Glycerinesterstruktur „angefochten" wird, was die 
nachfolgenden Reaktionsschritte erleichtern kann. Die Cholesterinester, gleich wie bei der basenkatalysierten Umesterung, haben auch im Falle der Verseifung ihre Widerstandsfähigkeit erwiesen. Hierbei könnte die Vollständigkeit der Umesterung eine Rolle spielen, bei höheren Konzentrationen $(>2 \mathrm{mg} / \mathrm{ml})$ wird wahrscheinlich die Reaktionszeit zu kurz dafür, um alle anwesenden Cholesterinester in ihre Methylester umzusetzen.

Um den Beweis zu erbringen, daß alle häufig vorkommenden langkettigen Lipidfettsäuren gleich abgespalten und verseift werden, $\mathrm{da} ß$ aber keine Isomerisierung vorkommt, haben wir ein menschliches Serum nach einer vorherigen dünnschichtchromatographischen Auftrennung in einzelne Lipidgruppen dreimal hydrolysiert (und mit $\mathrm{BF}_{3}-$ Methanol wieder verestert) (12) und dreimal mit $0,5 \mathrm{~mol} / 1$ Natriummethylat umgeestert (7). Der Anteil der einzelnen Säuren ((Mittelwert aus den drei Bestimmungen) wird in Tabelle 2 dargestellt. Die Ergebnisse zeigen klar, daß es auch im Falle von Polyensäuren zu keinem bedeutenden Verlust kommt.

Das hier dargestellte Verfahren verkürzt im Vergleich mit bisher bekannten Verfahren die Verseifungsdauer wesentlich und ermöglicht ohne Energiezufuhr von außen, auf „kaltem" Wege, eine gute Ausbeute von Fettsäuren. Schon drei Minuten nach Vermischen beider Reagenzien kann man mehr, als $\% / 10$ des eingesetzten Fettes als Fettsäuren extrahieren (falls die wäßrige Phase nicht angesäuert wird, extrahiert man den unverseiften Rückstand und die Fettsäuren verbleiben als Salze in der wäßrigen Phase). Die Verkürzung des Verseifungsvorganges bei der Bestimmung von Serumlipidgruppen durch die titrimetrische Methode nach Trout (11) wird im Labor den Vorteil bringen, da $ß$ man mehrere Proben ansetzen und bestimmen kann. Falls die Fettsäuren weiter zu einer gaschromatographischen Bestimmung herangezogen werden sollen, kann man sie wieder nach dem „Saponification-Reesterification" Verfahren (13) verestern.

\section{Literatur}

1. Zöllner, N. \&' 'Eberhagen, D. (1965), Untersuchung und Bestimmung der Lipoide im Blut, S. 146, Springer-Verlag,

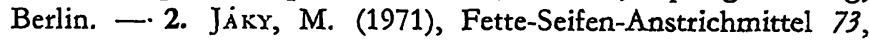
216-220. - 3. Glass, R. L. (1971), Lipids 6, 919-925. - 4 . Kuemmel, D. F. (1958), J. Amer. Oil Chemists' Soc. 35, $41-45$. 5. Klenk, E. \& Doss, M. (1966), Hoppe-Seylers Z. Physiol. Chem. 346, 296-298. - 6. Nichaman, M. Z., Sweeley, Ch. C. \& Otson, R. E. (1967), Amer. J .Clin. Nutr. 20, 1057-1069. - 7. HušEk, P. (1969), diese Z. 7, 627-630. -8. ANDERson, E. \&
Prerce, H. B. (1918), J. Phys. Chem. 22, 44-48. - 9. Peach, K. \& Tracey, M. V. (1955-56), Moderne Methoden der Pflanzenanalyse, Springer-Verlag, Berlin. - 10. Biochemica Test Combination, Boehringer Mannheim GmbH (Nr. 15989). 11. Trout, D. L., Esters, E. H. \& Friedierg, S. J. (1960), J. Lipid Res. 1, 199-202. - 12. Metcalfe, L. D. \& Schmitz, A. A. (1961), Anal. Chem. 33, 363-364. - 13. Metcalfe, L. D., Schmitz, A. A. \& Pelka, J. R. (1966), Anal. Chem. 38, 514-515.

\section{Petr Hušek}

Forschungsinstitut für Endokrinologie Abteilung Experimentelle Biochemie ČS-11694 Praha 1, Národní 8

CSSR 